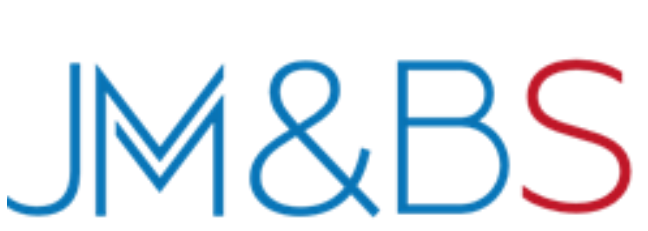

JM\&BS

Journal of

Management \& Business

Studies

\title{
Capital Intelectual y Universidades de Chile, un Modelo
} Teórico

\section{Intellectual Capital and Chilean Universities, a Theoretical Model}

\section{Claudio Inostroza-González ${ }^{1}$}

\section{Resumen}

El conocimiento se ha transformado en un activo corporativo, uno de los más importantes y la base principal para desarrollar una ventaja competitiva. El capital intelectual nos ayuda a medir los niveles de conocimiento y los intangibles acumulados por las empresas. Las universidades son un caso particular. Sabemos que son intensivas en conocimiento, lo que nos entrega un terreno fértil para el desarrollo y medición del capital intelectual. No obstante, pocos modelos consideran las particularidades de este tipo de instituciones. Considerando lo anterior, este trabajo propone un modelo teórico del capital intelectual que vincula las áreas de desarrollo de la academia con los componentes del capital intelectual, incorporando los dos niveles de desarrollo del conocimiento: explícito e implícito.

PALABRAS CLAVE: capital intelectual, conocimiento, capital social, capital humano, universidades.

\section{Abstract}

Knowledge has become into a corporate asset, one of the most important and the basis for developing a competitive advantage. Intellectual capital models help us to measure the levels of knowledge and intangibles accumulated by companies. We know that they are knowledge intensive. Despite the above, few models consider the particularities of this type of institutions. This paper proposes a model of intellectual capital that links the areas of development of the universities with the components of intellectual capital, incorporating the levels of knowledge development: explicit and implicit.

KEYWORDS: intellectual capital, knowledge, social capital, human capital, universities.

${ }^{1}$ Académico, Universidad Católica de la Santísima Concepción, Concepción, Chile. Master in Management Sciences, Universidad Adolfo Ibáñez. cinostroza@ucsc.cl. https://orcid.org/0000-0001-5193-5357. 
JM\&BS

\section{Introducción}

Uno de los cuestionamientos que el management se realiza, desde sus inicios, es el origen de la rentabilidad y por qué ésta difiere entre compañías (Rumelt et al, 1994). Es así como se plantean teorías y enfoques que permitan explicar de dónde proviene y cómo medirla (Mcgahan et al 1997; Rumelt, 1991; Khanna et al, 2001). Todo lo anterior, con la finalidad de lograr mejorar la rentabilidad, entendida ésta como la medida del desempeño.

En la actualidad, en distintos ámbitos del desarrollo económico y social, el énfasis está en lo "blando", lo intelectual. Es así como se desarrollan, por ejemplo, las llamadas empresas de la nueva economía, en donde el conocimiento es un elemento clave. Éstas, se desenvuelven en una era potenciada por el desarrollo de tecnologías de información y en donde la gestión de lo intangible es un punto importante.

Por otro lado, las compañías que habitualmente son foco de los estudios en este ámbito están vinculadas con la industria productiva o financiera. De la revisión literaria, se desprende que rara vez se han utilizado a las instituciones de educación como foco en este tipo de investigación (Bontis et al., 2000; Petty \& Guthrie, 2000; Pablos, 2002; Firer \& Williams, 2003; Cheng et al., 2005; Elbannany, 2008; Bontis, 2010; Serenko \& Bontis, 2013; Dumay \& Garanina, 2013). Sumado a lo anterior, durante los últimos años, en Chile, se ha puesto en cuestionamiento la capacidad de gestión de alguna de ellas que han debido cerrar definitivamente.

Considerando la naturaleza de este tipo de instituciones, es interesante aportar al desarrollo de modelos de capital intelectual. Sabemos que son intensivas en conocimiento, lo que nos entrega un terreno fértil para el desarrollo y medición del capital intelectual. Con un modelo que se adapte a la realidad de la educación superior, podemos evaluar el aporte que tiene este recurso intangible y estratégico en el desempeño de las universidades. Insertos en dicho contexto, podemos mencionar que existen muchas medidas del desempeño o "performance" de las compañías, la mayoría obtenida desde los sistemas de información que dan origen a los estados financieros (Mcgahan et al 1997; Rumelt, 1991; Khanna et al, 2001). Por tanto, existe la 
JM\&BS

necesidad de evaluar formas de mejorar el desempeño que no estén vinculadas directamente con el tradicional enfoque de los resultados financieros, que utiliza información contable en base a costo histórico y cuyo foco es la rentabilidad.

Sabemos que, en el ámbito de la educación, el conocimiento es un elemento clave y la acreditación un requisito fundamental. Sin embargo, en la revisión literaria no es común encontrar trabajos cuyo foco sea la evaluación del capital intelectual en las instituciones de educación superior. A través del planteamiento de este trabajo, se pretende ayudar a la gestión de las instituciones, aportando un modelo teórico de medición, en el contexto de la educación superior chilena, específicamente, las universidades.

El artículo parte con una revisión literaria de los conceptos y teorías más relevantes que han dado origen al capital intelectual, desde la visión basada en los recursos, para derivar en el análisis de la visión basada en el conocimiento y sus constructos asociados (capital intelectual, capital humano, capital estructural, capital de consumidores). Posteriormente, se deriva el modelo y sus componentes; culminando con las
Journal of

Management \& Business

Studies

conclusiones, limitaciones e implicancias.

\section{Revisión de la Literatura}

\subsection{Visión Basada en Recursos}

La visión basada en recursos (RBV) nace en el ámbito del management estratégico como una de las corrientes teóricas más importantes para explicar el desempeño de las firmas (Según Peng, Li Sun, Pinkham y Chen, 2009, éstas son: i) visión basada en la firma, ii) visión basada en recursos y iii) visión basada en las instituciones). No es una teoría del todo nueva (Hosskison et al 1999), ya que, en el año 1957 , Selznick hacía mención de la idea de "competencias distintas de una empresa" y Ansoff (1965) definía que la sinergia era generada por una combinación de capacidades o competencias que podemos atribuir a recursos intangibles de una firma. Sin embargo, la pionera sobre las bases de esta teoría es Edith Penrose, que en su libro del año 1959 "Theory of the Growth of the Firm", describió la empresa como un conjunto de recursos humanos y de otro tipo que, al interactuar, generan creación de conocimiento dentro de la firma, a través de la especialización, división del 
JM\&BS

trabajo, aprendizaje y trabajo en equipo (Kor \& Mahoney, 2004). La mirada de Penrose pone el foco en la creación de valor (Pitelis, 2004) y en defensa de la heterogeneidad de los recursos, como fuente del carácter único de una firma. La noción de que las empresas logran un único carácter o pueden diferenciarse en virtud de su heterogeneidad de recursos, es la base de RBV (Hosskison et al., 1999). Luego, a principios de la década de 1980, es Wernerfelt quien como investigador se dedica a desarrollar y definir este constructo, relacionándolo con las ventajas competitivas de las empresas. $\mathrm{Su}$ foco está puesto en el análisis de las empresas, desde el punto de vista de sus recursos, en lugar de lo referido a sus productos o servicios, lo que entregó una nueva perspectiva a las opciones estratégicas utilizadas hasta esa época. Wernerfelt (1984) plantea en su artículo, además, como desafíos a la teoría del RVB, el estudio de nuevas formas de implementar las estrategias con este enfoque $\mathrm{y}$, por supuesto, definición de metodologías que permitan identificar y medir los distintos tipos de recursos. Barney $(1991,2001)$ plantea las características de los recursos, para que éstos se sostengan como ventaja competitiva. Muchas de estas características son difíciles de mantener a través de los activos tangibles tradicionales, pero si se puede ver claramente que, los intangibles como el conocimiento adquirido, tienen más posibilidades de cumplirlas. Dos características fundamentales son la baja probabilidad de ser imitado, es decir, la capacidad de protegerlos de copia por parte de los competidores; y que no sean transferibles, lo que implica que los competidores no posean los recursos equivalentes para sustituir un recurso por otro similar que les permita implementar la misma estrategia.

\subsection{Visión basada en el conocimiento}

Hoy día en el mundo de los negocios, el conocimiento se ha transformado en un activo corporativo, el más importante de todos y la base principal para una ventaja competitiva (Marr \& Spender, 2004). Algunos de los aspectos relevantes que contribuyen a esta tendencia son, por ejemplo, que el sector terciario ha experimentado un aumento importante a nivel mundial. En la década de los noventa, alrededor del 90\% de los puestos de trabajo, en América Latina, surgen del sector de 
JM\&BS

servicios (Weller, 2004). Por otra parte, los consumidores más informados demandan diferenciación e innovación o que el incremento de la globalización permite el acceso a recursos tangibles en cualquier parte, con lo que estos activos han perdido su carácter de estratégicos (Marr, 2004).

Los trabajos basados en el conocimiento han debatido si el origen de este y si el nuevo conocimiento con valor agregado es individual o colectivo. (Felin \& Hesterly, 2007). Kogut y Zander apoyan una perspectiva colectivista, ya que una empresa existe dado que genera acción social estructurada mediante principios organizadores que no son reducibles a los individuos. La empresa sería una comunidad social especializada en la transferencia de conocimiento (Kogut \& Zander, 1996). Este enfoque, sólo permite variables colectivas como explicaciones de los resultados tales como la cultura, la comunidad, la rutina y el medio ambiente (Felin \& Hestley, 2007).

El otro enfoque da énfasis al individuo como actor principal en la creación del conocimiento y el principal depósito del conocimiento, ya que todo
Journal of

Management \& Business

Studies el aprendizaje organizacional tendría lugar dentro de las cabezas humanas. Esto implica que una organización aprende con el conocimiento de sus miembros o incorporando nuevos miembros que tengan el conocimiento que la organización necesita (Felin \& Hestley, 2007; Simon, 1991).

Existe también la distinción entre conocimiento tácito y explícito, que fue desarrollada por Polanyi e incorporada por Nelson \& Winter (1982), junto con la teoría de evolución de la firma. Nonaka y Takeuchi (1995) hacen de la interacción entre los dos tipos de conocimiento, la base para el proceso de creación del conocimiento organizacional. Esta clasificación permite activar los mecanismos de adaptación.

El conocimiento tácito permanece en un nivel "inconsciente", se encuentra desarticulado y es difícil de expresar. El conocimiento implícito, para efectos de este trabajo, es el tácito que se puede externalizar. Éste resulta de los hábitos acumulados, intuición, costumbres, creencias, valores. Generalmente, es personal o de un grupo bien cohesionado y opera de forma mecánica, dado que está incorporado en 


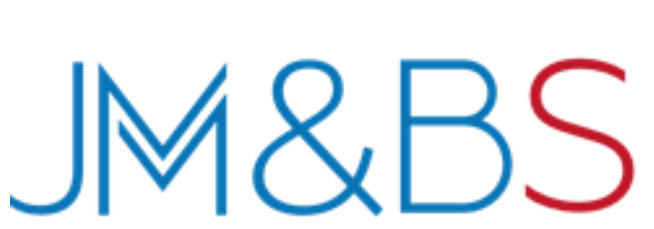

el individuo o el grupo. Tiene un componente importante de experiencia y aprendizajes personales. Tiene componentes asociados, tanto a habilidades técnicas, como cognitivas (Vega-Muñoz, 2010).

El conocimiento explícito tiene forma y es sistemático, por lo que es más fácil de compartir. Este se puede encontrar almacenado en documentos, bases de datos o manuales. Es aquél que podemos verbalizar y envasar para poder transmitir. Este conocimiento puede obtenerse desde el conocimiento implícito. Esa transferencia se realiza mediante su aplicación o a través del cumplimiento reglas de aplicación de criterios.

En este contexto emerge una teoría que caracteriza al conocimiento

\section{Journal of}

Management \& Business

Studies

como un recurso estratégico, la llamada "knowledge-based theory of the firm" (Grant, 1996). Esta visión considera al conocimiento como el recurso primario de una empresa, que está inserto en la cultura organizacional y en la identidad, rutinas y sistemas, así como en las mentes de los individuos. El conocimiento, como recurso, puede facilitar la comprensión de las presunciones básicas importadas de las teorías vinculadas a la dependencia de recursos como el "Resource Based View" (Alajmi, 2008).

La siguiente figura resume los enfoques teóricos que sustentan el constructo de capital intelectual.

Figura 1. Enfoques teóricos que sustentan el capital intelectual

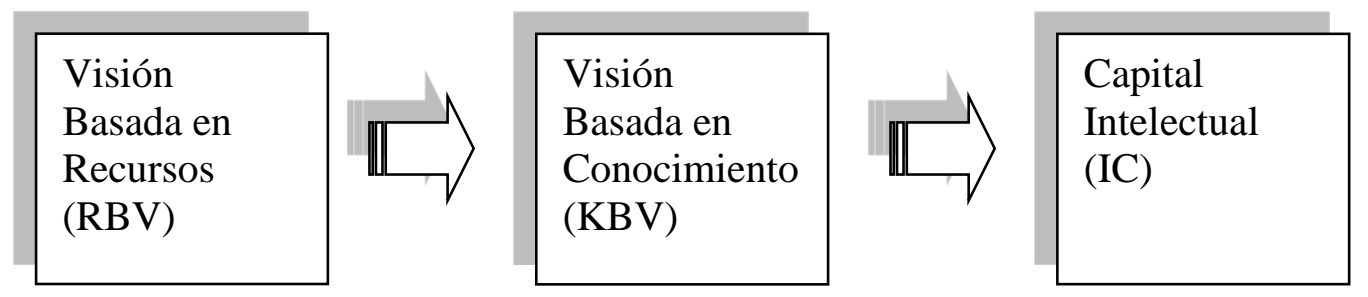

Fuente: Elaboración propia. 
JM\&BS

\subsection{El capital intelectual}

Los contadores y gerentes de finanzas están interesados en cómo medir el capital intelectual y llevarlo al balance, los informáticos quieren incorporarlo en los sistemas, los psicólogos quieren trabajar las mentes en base a él, los gerentes de recursos humanos quieren medirlo, evaluarlo y asegurarse de crearlo, etc. (Bontis 1999). Este concepto intriga a varios grupos de interés en la sociedad de nuestros días, dada su naturaleza intangible y compleja.

\section{Journal of}

Management \& Business

Studies
Kristandl \& Bontis (2007) realizan una definición de este concepto basándose en la teoría del recursos y capacidades, en base a las denominadas características VRIN (valioso, raro, inimitable, no sustituible). Aquéllos que cumplen con las características mencionadas, son subclasificados en tangibles e intangibles. Luego, los intangibles, en donde está el foco de este estudio; están constituidos por el capital intelectual, que, a su vez, está integrado por capital humano y capital estructural.

Figura 2: Intangibles y capital intelectual.

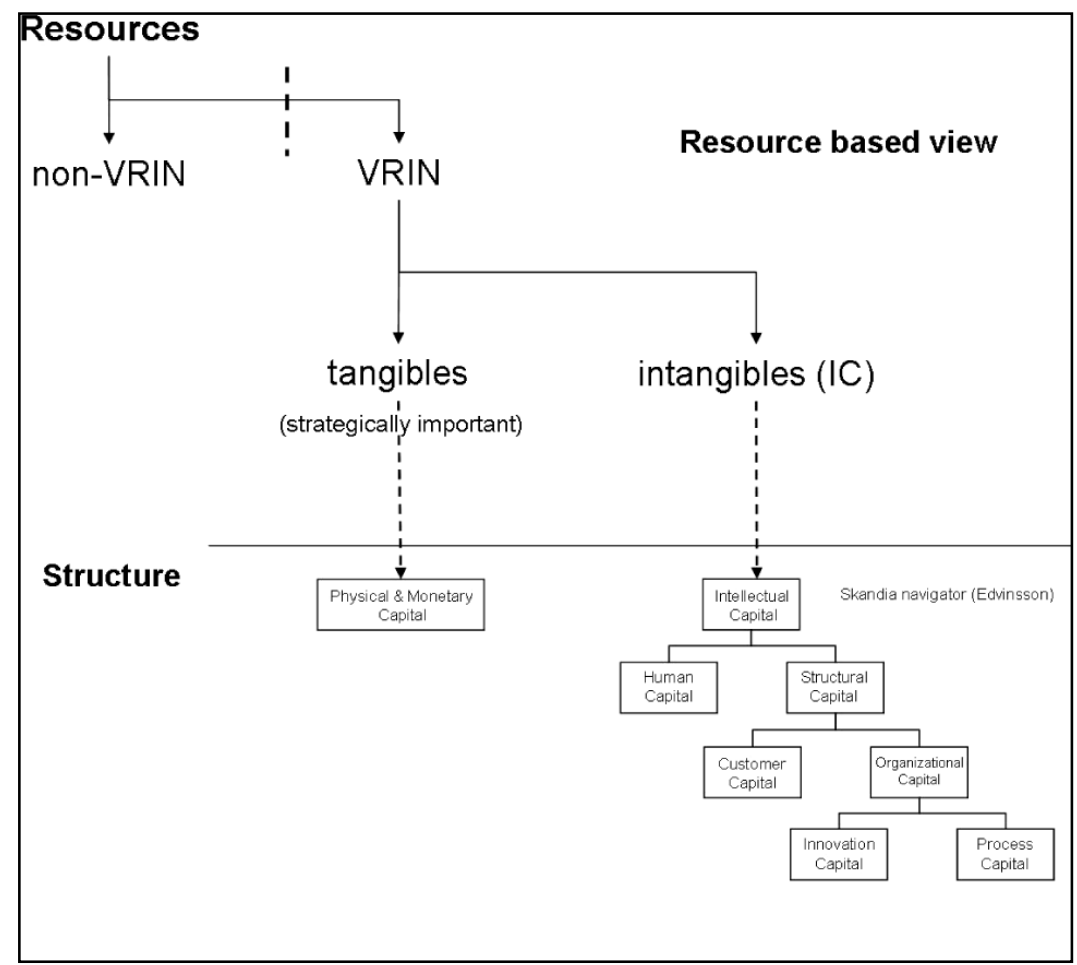

Fuente: Kristandl y Bontis (2007) 
JM\&BS

A mediados de la década de los noventa, cuando se hablaba de intangibles y específicamente de capital intelectual, se utilizaba la definición Edvinsson y Malone (1997), la que podemos resumir en la siguiente ecuación: $($ Capital Intelectual $=$ Valor de mercado - Valor libro). Esta era una definición simplista y muy general.

Stewart (1997), define el capital intelectual como el material intelectual, el conocimiento, información, propiedad intelectual, experiencia que se puede utilizar para crear riqueza. La principal contribución de Stewart estaba en la definición del capital intelectual y en el reconocimiento de la dificultad para medirlo. El capital intelectual significa más que sólo "el intelecto puro" sino que "la acción intelectual". En ese sentido, el capital intelectual no sólo es un activo intangible estático, es un proceso
Journal of

Management \& Business

Studies

ideológico; un medio para un fin (Bontis, 1998). Youndt et al (2004) definen este constructo como la suma de todo el conocimiento que una organización es capaz de influenciar en el proceso de dirigir el negocio hacia ganar ventajas competitivas.

Puede ser útil, considerar el capital intelectual como la unidad de acción a través de flujos de aprendizaje. Sin embargo, el capital intelectual puede no siempre ser enseñado a través de la educación y la formación. El conocimiento más valioso en una organización a menudo puede no ser transmitido (Levitt, 1991). Este constructo se puede definir, según Bontis, de acuerdo con la figura 3, a través de sus componentes: capital humano, capital estructural y capital de consumidores/clientes. 


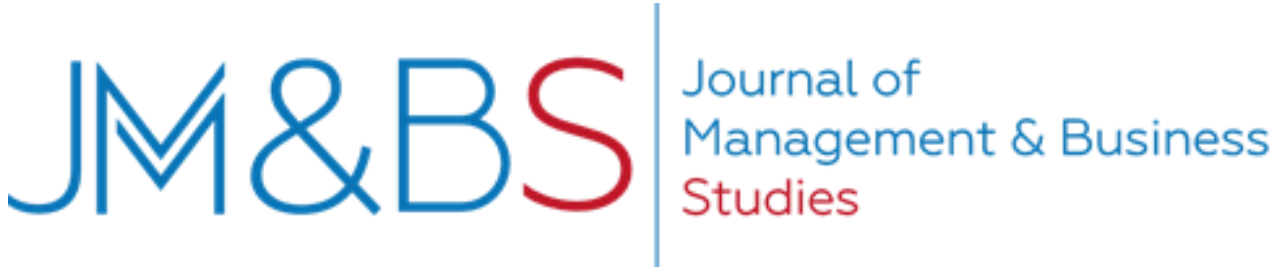

Figura 3. Conceptualización de capital intelectual,

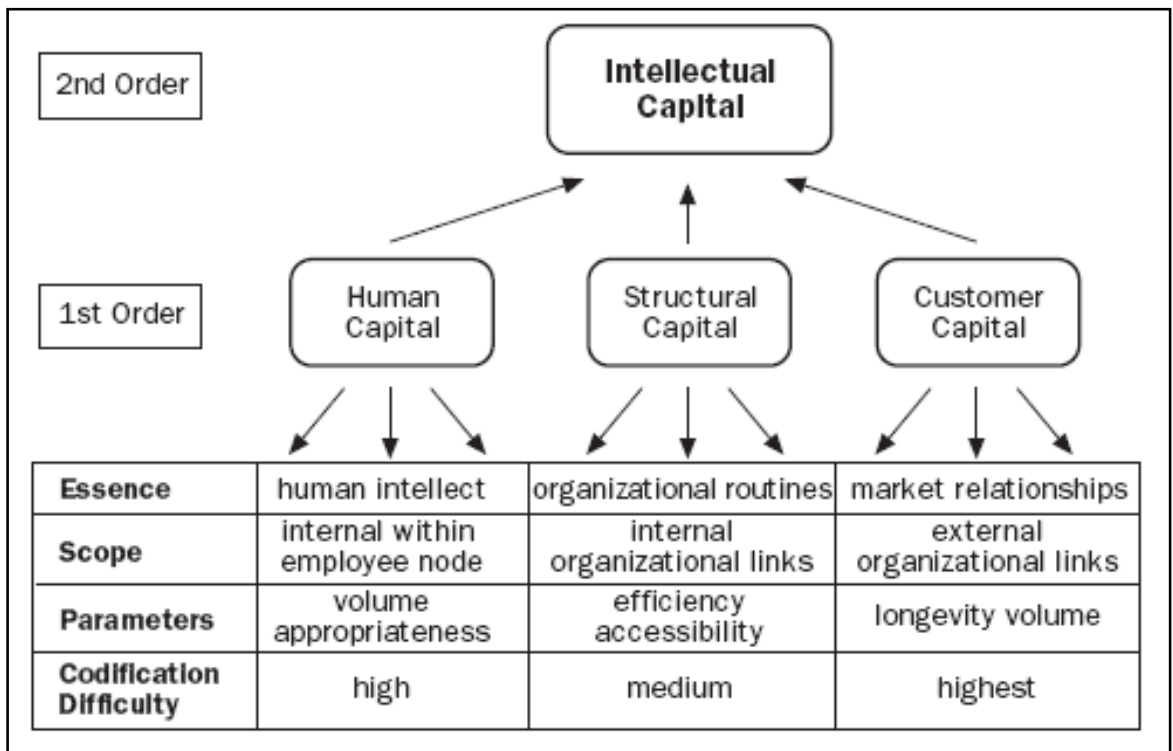

Fuente: Bontis (1998).

Dada esta definición, consideraremos a la organización como un proceso productivo que recibe desde el ambiente insumos de dos tipos: materiales e información y luego produce bienes o servicios tangibles y de información, que vuelven al ambiente. Cada uno de los componentes de primer orden, se definen, según el autor como:

Capital humano: Se define a un nivel individual como la combinación de cuatro factores: i) herencia genética; ii) educación; iii) experiencia y iv) actitudes acerca de la vida y los negocios. El capital humano es importante porque se trata de una fuente de innovación y renovación estratégica. Los integrantes de una organización poseen conocimiento tácito individual (Nelson y Winter, 1982).

Capital estructural: Se refiere a los mecanismos y las estructuras de la organización que pueden dar soporte a los empleados en su búsqueda del rendimiento intelectual óptimo $y$, por lo tanto, el rendimiento global de la firma. Si el capital estructural es fuerte dentro de la empresa, existirá una cultura de apoyo a los individuos, potenciando lo positivo y evitando el miedo al fracaso, lo que permite obtener su potencial máximo.

Capital de consumidores: el conocimiento relativo a los canales de 
JM\&BS

comercialización y las relaciones con los

consumidores son el elemento clave de este ítem. Estos intangibles incluyen el conocimiento inserto en los clientes, los proveedores, el gobierno o asociaciones relacionadas con la industria. Éste, se constituye en el más complejo de los tres componentes del capital estructural, ya que es el más externo al núcleo de la organización.

Cuando se discute el aprendizaje individual y colectivo, se suele decir que son las personas, quienes poseen $\mathrm{y}$ generan el conocimiento. Queda en segundo plano la propuesta de que tanto los individuos como las organizaciones aprenden. El argumento contrario propone que las organizaciones también aprenden, en la medida que sus miembros sean maleables, lo que implica que su individualidad se vea influenciada por la identidad de la organización (Huber, 1991; Spender, 1996).

La investigación en capital intelectual se ha desarrollado en las últimas décadas, pero principalmente con foco en las necesidades de los ejecutivos y profesionales en negocios, no desde la academia; por lo que el campo está dominado por un número relativamente pequeño de autores
(Bontis, 2002; Anddriessen, 2004). Su origen, se puede vincular con la necesidad de "medir"; lo que podríamos resumir en la frase "no puedes manejar lo que no puedes medir" (Stewart, 2001; Roos et al., 1997).

Los sistemas que miden capital intelectual incorporan listas de indicadores sin priorización, lo que hace que sea imposible para los gerentes evaluar el "trade-off" de las decisiones o la interacción entre las variables (Roos et al., 1997). Además, suele darse énfasis a los indicadores que aseguren la riqueza de los accionistas, los que tradicionalmente se basan en ganancias derivadas de la contabilidad.

\section{Acreditación}

de

\section{Universidades}

Las universidades, son instituciones dedicadas a la educación terciaria y que mantienen, entre sus recursos, una variedad de activos físicos, financieros e intangibles, que deben ser gestionados de forma eficiente, con el fin de cumplir su estrategia, la que incluye las áreas básicas del desarrollo académico: docencia, investigación gestión y vinculación con el medio. Estas áreas se vinculan, además, con los 
JM\&BS

criterios utilizados para acreditar a las universidades, en Chile. Esta es una medida de desempeño considerada relevante y que permite el acceso a beneficios estatales. La acreditación está incluida como un objetivo dentro de la estrategia de las universidades chilenas.

La Comisión Nacional de Acreditación de Chile, es un organismo público, autónomo, cuya función es verificar y promover la calidad de las Universidades. La acreditación forma parte de los sistemas de aseguramiento de la calidad y gestión de este tipo de instituciones y es requisito para una serie de beneficios por parte del Estado. A través de la acreditación, se busca verificar que las casas de estudio cumplan los requerimientos básicos propios de una institución de educación superior; de manera de avanzar eficaz y eficientemente hacia el logro de sus propósitos, lo que implica evaluar el proceso y ajustar cuando corresponda.

Las áreas de acreditación de las Universidades, según la Comisión Nacional de Acreditación, se definen a continuación:

Gestión: por gestión institucional se entiende el conjunto de políticas y mecanismos destinados a
Journal of

Management \& Business

Studies

organizar las acciones y recursos de la institución, en función de sus propósitos y fines declarados. Considera la organización y estructura institucional, el sistema de gobierno y la administración de recursos humanos, materiales y financieros.

Docencia: en el ámbito de la docencia, implica asegurar la calidad de la formación de pregrado y postgrado, con especial énfasis en los aspectos relacionados con el diseño y aprobación de los programas ofrecidos; con su implementación y seguimiento; y con un análisis de sus resultados y los mecanismos para revisar y modificar el currículo, la organización de los programas, los métodos pedagógicos $\mathrm{y}$ otros aspectos que afecten la calidad de la formación entregada.

Investigación: por investigación se entienden las actividades sistemáticas de búsqueda de nuevo conocimiento, de alto nivel en rigurosidad de los proyectos y su contribución, que impactan sustantivamente en la disciplina, tema o área a la que pertenecen. Sus resultados se expresan en publicaciones $\mathrm{o}$ en patentes.

Vinculación con el medio: la vinculación con el medio, en el marco 
JM\&BS

del proceso de acreditación institucional, se refiere al conjunto de nexos establecidos con el medio disciplinario, artístico, tecnológico, productivo o profesional, con el fin de mejorar el desempeño de las funciones institucionales, de facilitar el desarrollo académico y profesional de los miembros de la institución y su actualización o perfeccionamiento, o de cumplir con los objetivos institucionales.

\section{Propuesta de modelo}

Las Universidades, son organizaciones con actividades y características particulares. El desarrollo de sus actividades y el foco de su quehacer se vinculan con la generación y difusión del conocimiento. Las organizaciones aprenden, en la medida que sus miembros sean maleables, lo que implica que su individualidad se vea influenciada por la identidad de la organización (Huber, 1991; Spender, 1996). Estas actividades (docencia, investigación, gestión y vinculación), se asocian con los componentes del capital intelectual (humano, estructural y relacional). El enfoque de capital intelectual involucra la generación y transferencia de conocimiento, lo que
Journal of

Management \& Business

Studies

nos muestra la existencia de un nexo directo entre este enfoque y las universidades. A pesar de lo anterior, no existe variedad en modelos y propuestas que contribuyan al desarrollo del capital intelectual; que potencien el desarrollo de sus componentes o que permitan monitorear la gestión de sus elementos y su contribución al cumplimiento de los objetivos de una organización de este tipo.

Incorporando la distinción entre conocimiento implícito y explícito, que fue desarrollada por Polanyi e incorporada por Nelson \& Winter (1982) y el modelo tradicional de Bontis (1998), desarrollamos un modelo cuyos componentes se detallan a continuación:

\subsection{Dimensión: capital humano}

Lo definiremos como el conocimiento que los recursos humanos de la organización pueden llevarse con ellos cuando se van de la universidad, es decir, son un tipo de conocimiento que adquieren las personas. Este tipo de conocimiento, dado su origen, se desarrolla en el nivel de implícito. Los integrantes de una organización poseen conocimiento tácito individual (Nelson y Winter, 1982). Este conocimiento 
JM\&BS

alimenta la dimensión "docente", una de las principales actividades del quehacer universitario a través de su dimensión implícita. La formación inicial, capacitación y experiencia de cada individuo, generan conocimiento implícito que moldean la forma de enseñar a los demás. Proponemos, además, que existe un flujo que conecta esta dimensión con la estructural, que es la que da soporte a las personas. La dimensión explícita del capital estructural alimenta la dimensión implícita del capital humano.

Proposición 1: Existe una relación positiva entre el capital humano y los niveles de acreditación de las universidades

\subsection{Dimensión: capital estructural}

Lo definiremos como el stock de infraestructura que posea la universidad, necesaria para coordinar esfuerzos y convertir el conocimiento en productos, pero también la forma de hacerlo. El principal desafío de esta dimensión es hacer explícito el conocimiento implícito que hay en una organización. Por tanto, conviven claramente en esta dimensión ambos enfoques del conocimiento: implícito y explícito. Proponemos que
Journal of

Management \& Business

Studies

existe un flujo de conocimiento que fluye desde la dimensión estructural hacía el capital humano. Es habitual que la universidad entregue formación, recursos e infraestructura a los académicos, lo que les permite generar nuevo conocimiento y potenciar el conocimiento implícito previo. La dimensión implícita del capital estructural recibe, también flujo de conocimiento desde el capital relacional. Proposición 2: Existe una relación positiva entre el capital estructural y los niveles de acreditación de las universidades.

Proposición 2b: Existe una relación positiva entre la dimensión explícita del capital estructural y la dimensión implícita del capital humano.

\subsection{Dimensión: capital relacional}

Las universidades forman parte de la sociedad y deben estar integradas a sus comunidades. Por lo tanto, definiremos esta dimensión como la pertenencia a redes que la vinculan con los stakeholders. La universidad obtiene de estas redes conocimiento que va a integrar a la estructura de su organización con el fin de mejorar y lograr los objetivos planteados en la 
JM\&BS

estrategia. El entorno y el medio externo proveen del "benchmarking", que la institución absorbe e internaliza al transferirlo al capital estructural. El conocimiento extraído de estos nexos con el exterior debe ser explícitamente incorporados, de modo de aprovecharlos en el resto de las áreas de la organización. Por lo anterior, proponemos que esta dimensión entrega

\section{Journal of}

Management \& Business

Studies un flujo de conocimiento explícito al capital estructural.

Proposición 3: Existe una relación positiva entre el capital relacional y los niveles de acreditación de las universidades.

Proposición 3b: Existe una relación positiva entre la dimensión explícita del capital relacional y la dimensión implícita del capital estructural.

Figura 4: Modelo teórico de capital intelectual para Universidades

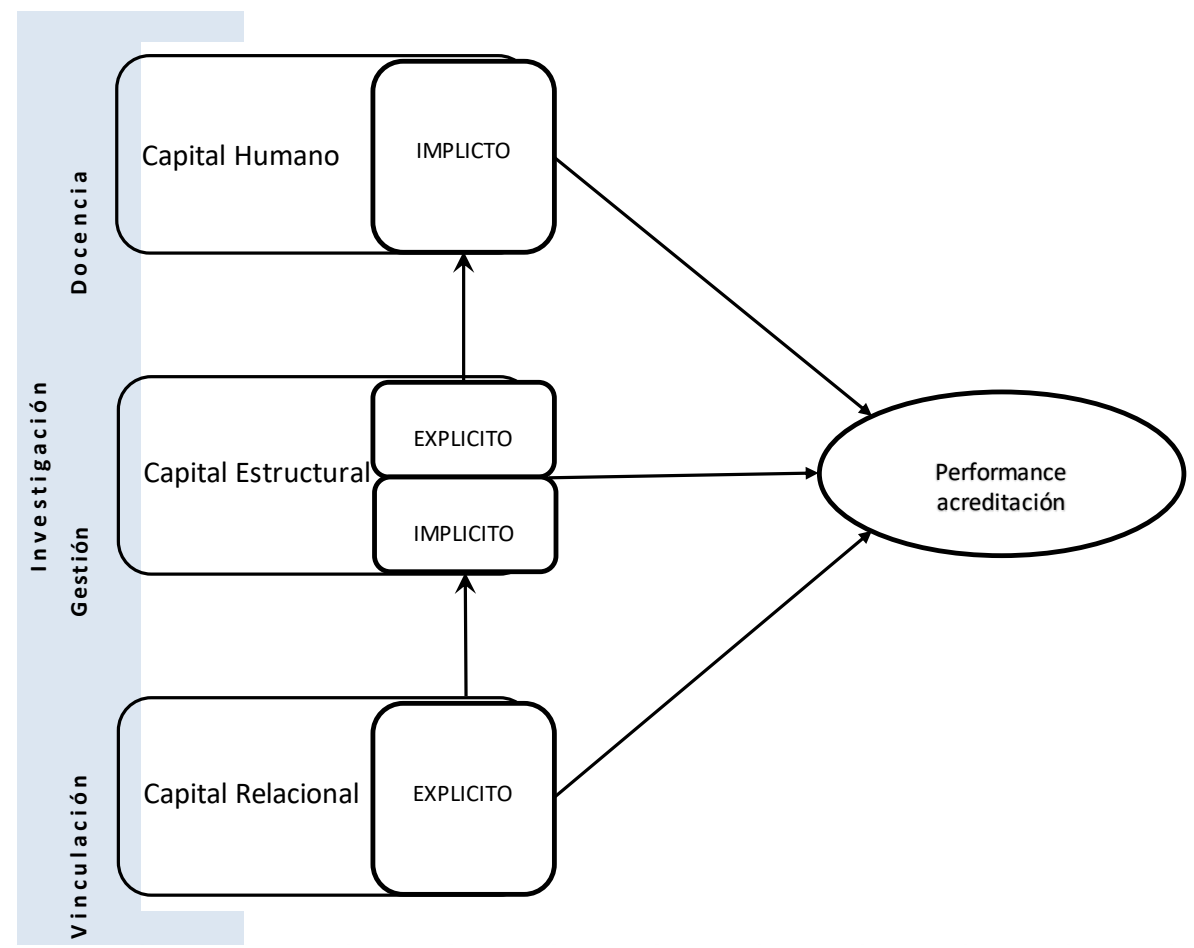

Fuente: Elaboración propia. 
JM\&BS

5. Conclusiones, limitaciones

e implicancias

A pesar de ser un tema que ha tenido desarrollo en las últimas décadas, la literatura ha explorado poco el capital intelectual en empresas públicas, sin fines de lucro $\mathrm{u}$ otras similares. $\mathrm{La}$ empresa "tradicional", tiene una forma de gestión y objetivos estratégicos que difieren de instituciones como las universidades. Estas últimas invierten principalmente en recursos humanos e investigación; y tienen como preocupación la producción y difusión del conocimiento. No obstante, existen instrumentos muy limitados para medir y gestionar el capital intelectual en ese contexto.

Hoy en día, una institución de educación superior debe contar con las certificaciones y acreditaciones necesarias que permitan a los estudiantes y público en general, confiar en la institución y en su calidad. Junto con los objetivos de generación y difusión del conocimiento, se cruzan aquellas áreas que son foco de acreditación: docencia, gestión, investigación y vinculación con el medio.

Las áreas de acreditación se vinculan directamente con el quehacer de la universidad y con los componentes del capital intelectual. El capital humano involucra las habilidades $\mathrm{y}$ conocimientos que se desarrollan con la formación y en el ejercicio de la docencia. El capital estructural incluye los recursos que dan soporte a la gestión universitaria. El capital relacional absorbe el conocimiento proveniente de la vinculación que la universidad desarrolla con su entorno. Por último, la investigación moviliza recursos humanos, de soporte y relacionales.

El vincular los tipos de conocimiento explícito-implícito en el modelo, es importante, debido a que los flujos de conocimiento se transfieren en distintos estados. Esto permite identificar el tipo de conocimiento que más se vincula con cada área de desarrollo y cómo fluye al resto de la universidad. Además, permite orientar los flujos de transferencia y apropiación del conocimiento en las distintas áreas. Este mecanismo de transferencia y adaptación del conocimiento está en la base de la evolución de una organización.

El enfoque propuesto permite integrar algunos componentes que enriquecen el modelo básico de capital 
JM\&BS

intelectual, entregando una herramienta mejorada para la gestión del conocimiento en las universidades. El modelo permite evaluar qué componente del capital intelectual tiene mayor influencia sobre los niveles de acreditación. Se puede comparar si los componentes que más contribuyen coinciden con las áreas de desarrollo prioritarias para la universidad. De ese ejercicio, pueden obtenerse información que genere ajustes en la estructura o en la estrategia.

\section{Journal of}

Management \& Business

Studies
El modelo a pesar de basarse en la realidad chilena puede ser replicado en otros contextos. Con un modelo que se adapte a la realidad de la educación superior, se provee de una herramienta para evaluar y gestionar el aporte que tienen los recursos estratégicos de este tipo, en el desempeño de las universidades $y$ en el nivel de la acreditación. Debemos considerar la propuesta como un modelo teórico que necesita ser probado empíricamente para verificar los supuestos y la relación entre las variables.

\section{Referencias:}

Andriessen, D. (2004). IC valuation and measurement: classifying the state of the art.

Journal of Intellectual Capital, 5(2), 230-242.

Alajmi, Bibi (2008). Understanding Knowledge-Sharing Behavior: A Theoretical Framework. Knowledge management in organizations course. Doctoral Program in Communication, Information and Library Science and Media Studies. Rutgers-The Satate University of New Jersey.

Ansoff H. I. (1965). Corporate Strategy: An Analytic Approach to Business Policy For Growth and Expansion, McGraw-Hill, New York.

Barney, J. (1991). Firm Resources and Sustained Competitive Advantage. Journal of Management, 17(1), 99-120.

Barney, J. B., Ketchen, D. J., \& Wright, M. (2011). The Future of Resource-Based Theory: Revitalization or Decline? Journal of Management, 37(5), 1299-1315.

Barney, J. B. (2001). Resource-based theories of competitive advantage : A ten- year retrospective on the resource-based view. Journal of Management, 27, 643-650.

Bontis, N. (1998). Intellectual capital: an exploratory study that develops measures and models. Management Decision, 36(2), 63-76. 


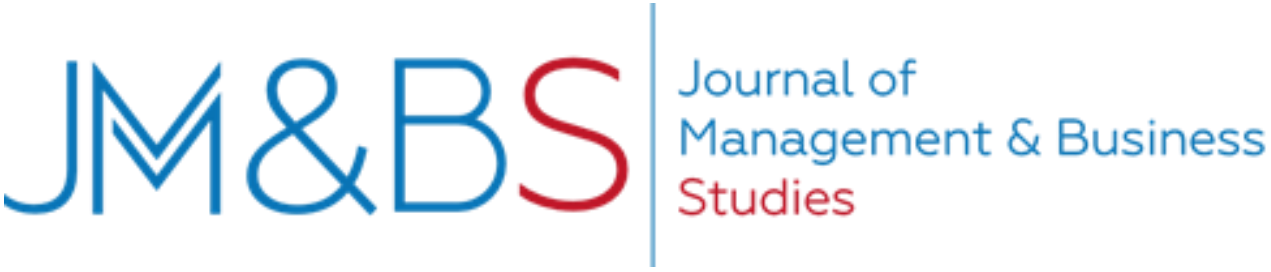

Bontis, N. (1999). Managing organizational knowledge by diagnosing intellectual capital: framing and advancing the state of the field. Int. J. Technology Management, 18.

Bontis, N., Chua, W., \& Richardson, S. (2000). Intellectual capital and business performance in Malaysian industries industries. Journal of Intellectual Capital, 1(1), 85100.

Bontis, N. (2010). Assessing knowledge assets: a review of the models used to measure intellectual capital. International Journal of Management Reviews, 3(1), 41-60.

Chen, M., Cheng, S., \& Hwang, Y. (2005). An empirical investigation of the relationship between intellectual capital and firms' market value and financial performance. Journal of Intellectual Capital, 6(2), 159-176.

Dumay, J., \& Garanina, T. (2013). Intellectual capital research: a critical examination of the third stage. Journal of Intellectual Capital, 14(1), 10-25.

Edvinsson, L. \& Malone, M.S. (1997), Intellectual Capital: Realizing your Company’s True Value by Finding Its Hidden Brainpower, HarperCollins Publishers, New York, NY. El-bannany, M. (2008). A study of determinants of intellectual capital performance in banks : the UK case. Journal of Intellectual Capital, 9(3), 487-498.

Felin, T., \& Hesterly, W. S. (2007). The knowledge-based view, nested heterogeneity , and new value creation : philosophical considerations on the locus of knowledge, 32(1), $195-218$.

Firer, S., \& Williams, S. M. (2003). Intellectual capital and traditional measures of corporate performance. Journal of Intellectual Capital, 4(3), 348-360.

Grant, R. M. (1996). Toward a knowledge based view of the firm. Strategic Management Journal, 17(winter), 109-122.

Guthrie, J. (2001). The management, measurement and the reporting of intellectual capital. Journal of Intellectual Capital, 2(1), 27-41.

Huber, G. (1991). 'Organizational learning: The contributing processes and the literatures', Organization Science, 2, pp.88-115.

Khanna, T., \& Rivkin, J. (2001). Estimating the performance effects of business groups in emerging markets. Strategic Management Journal, 22(July 2000), 45-74.

Kristandl, G., \& Bontis, N. (2007). Constructing a definition for intangibles using the resource based view of the firm. Management Decision, 45(9), 1510-1524. 


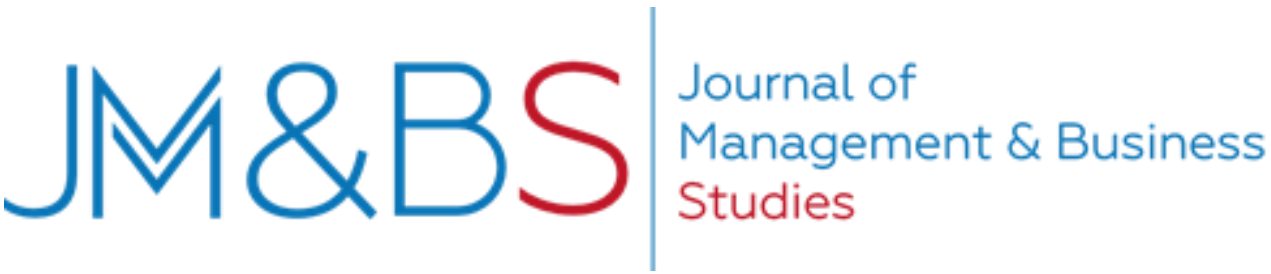

Kogut, B., \& Zander, U. (1993). Knowledge of the firm and the evolutionary theory of the multinational corporation. Journal of International Business Studies, 24(4), 625-645. Kogut, B., and Zander, U. (1996). What firms do? Coordination, identity, and learning, Organization Science (7:5), pp. 502-518.

Kor, Y. Y., \& Mahoney, J. T. (2004). Edith Penrose' s ( 1959 ) Contributions to the Resource-based View of Strategic Management, (January).

Levitt, T. (1991), Marketing Imagination, The Free Press, New York, NY

Marr, B., \& Spender, J.C. (2004). Measuring knowledge assets - implications of the knowledge economy for performance measurement. Measuring Business Excellence, $8(1), 18-27$.

Mcgahan, A. M., \& Porter, M. E. (1997). How much does industry matter, really? Strategic Management Journal, 18 (Special issue), 15-30.

Nelson, R.R. and Winter, S.G. (1982), An Evolutionary Theory of Economic Change, Belknap Press, Cambridge, MA.

Nonaka, I., Y Takeuchi, H., (1994). La organización creadora de conocimiento. Edición en español de 1999. Oxford University Press.

Pablos, P. (2002). Evidence of intellectual capital measurement from Asia, Europe and the Middle East. Journal of Intellectual Capital, 3(3), 287-302.

Penrose, E. (1959). Introduction: The Purpose of the Study. The Nature of the Argument. In The Theory of the Growth of the Firm (Third., pp. 1-8). Oxford University Press.

Peng, M. W., Sun, S. L., Pinkham, B., \& Chen, H. (2009). The Institution-Based View as a Third Leg for a Strategy Tripod. Academy of Management Perspectives, 63-82.

Petty, R., \& Guthrie, J. (2000). Intellectual capital literature review, measurement, reporting and management. Journal of Intellectual Capital, 1(2), 155-176.

Pitelis, C. N. (2004). Edith Penrose and the resource-based view of (international) business strategy. International Business Review, 13(4), 523-532.

Polanyi, M. (1967). The Tacit Knowledge Dimension. London: Routledge \& Kegan Paul Roos, G., Roos, J., Dragonetti, N. and Edvinsson, L. (1997), Intellectual Capital; Navigating in the New Business Landscape, New York University Press, New York, NY. Rumelt, R. P., Schendel, D. E., \& Teece, D. J. (1994). Fundamental Issues in Strategy (pp. 9-47). Boston MA.: Harvard Bussines School Press. 
ISSN 2452-5340

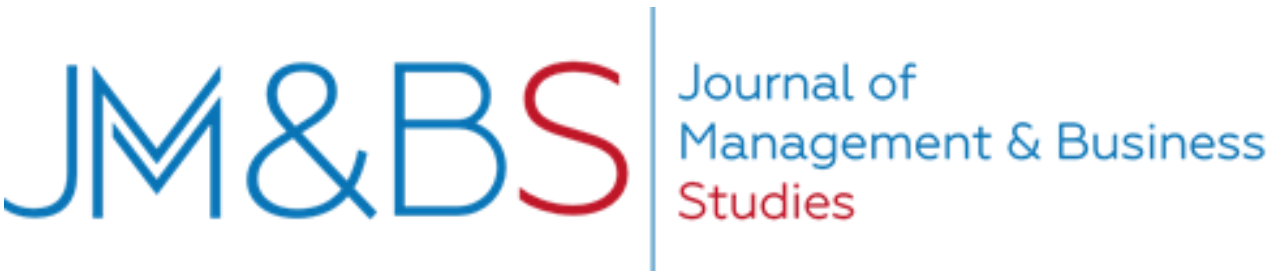

Rumelt, R. P. (1991). How much does industry matter?. Strategic Management Journal, 12(3), 167-185.

Serenko, A., \& Bontis, N. (2013). Investigating the current state and impact of the intellectual capital academic discipline. Journal of Intellectual Capital, 14(4), 476-500. Spender, J.-C. (1996). Making Knowledge the Basis of a Dynamic Theory of the firm. Strategic Management Journal, 17, 45-62.

Stewart, T.A. (1997), Intellectual Capital: The NewWealth of Organizations, doubleday/Currency, New York, NY.

Stewart, T.A. (2001), The Wealth of Knowledge: Intellectual Capital and the Twentyfirst Century Organization, Doubleday/Currency, New York, NY.

Vega-Muñoz, A. (2010). Redes de Innovación y Gestión del Conocimiento. Latin American Journal of International Affairs, 2(2), 10-21. Recuperado de: https://www.lajia.net/lajia/article/view/10.

Weller, J. (2004). El empleo terciario en América Latina: entre la modernidad y la sobrevivencia. Revista de la CEPAL 84. Diciembre 2004.

Wernerfelt, B. (1984). A Resource-based View of the Firm. Strategic Management Journal, 5(2), 171-180.

Youndt, M. A., Subramaniam, M., \& Snell, S. A. (2004). Intellectual Capital Profiles: An Examination of Investments and Returns. Journal of Management Studies, 41(2). 